\title{
A Recurrent Neural Network for Solving a Class of General Variational Inequalities
}

\author{
Xiaolin Hu, Student Member, IEEE, and Jun Wang, Fellow, IEEE
}

\begin{abstract}
This paper presents a recurrent neural-network model for solving a special class of general variational inequalities (GVIs), which includes classical VIs as special cases. It is proved that the proposed neural network (NN) for solving this class of GVIs can be globally convergent, globally asymptotically stable, and globally exponentially stable under different conditions. The proposed NN can be viewed as a modified version of the general projection NN existing in the literature. Several numerical examples are provided to demonstrate the effectiveness and performance of the proposed $\mathrm{NN}$.
\end{abstract}

Index Terms-General projection neural network (GPNN), general variational inequalities (GVIs), global asymptotic stability, global exponential stability, recurrent neural network.

\section{INTRODUCTION}

D URING the past two decades, neural networks (NNs) have found numerous applications such as classification, pattern recognition, associate memory system, optimization, and control. Specifically, recurrent NNs have served as efficient alternatives for conventional iterative numerical methods in dealing with many computational problems. For instance, there have been many successful applications of recurrent NNs for solving linear algebraic equations, matrix algebra problems, linear and quadratic programming problems, etc. (see [1]-[4] and references therein for example). Among them, an important class of problems refers to the variational inequalities (VIs).

VI was introduced in the early 1960s, and it has enjoyed a vigorous growth since then [5], [6]. It has many applications such as the analysis of piecewise-linear resistive circuits, bimatrix equilibrium points problem, economic equilibrium modeling, traffic network equilibrium modeling, structural analysis, and so on. A survey of results and applications can be found in [7]. In recent years, several recurrent NNs were proposed for solving VIs. In [8], a recurrent NN was presented for solving linear VIs. At almost the same time, another neural-network model for solving linear VIs was invented in [9]. In [10]-[13], a projection NN was developed for solving nonlinear VIs and related optimization problems with box or sphere constraints. More recently, two NNs capable of solving nonlinear VIs with general constraints were proposed in [14]-[16], which represent the state of the art in this area.

Manuscript received March 31, 2006; revised June 20, 2006. This work was supported by the Hong Kong Research Grants Council under Grant CUHK4165/03E. This paper was recommended by Associate Editor P. De Wilde.

The authors are with the Department of Automation and Computer-Aided Engineering, The Chinese University of Hong Kong, Sha Tin, Hong Kong.

Digital Object Identifier 10.1109/TSMCB.2006.886166
To date, various extensions of VI have also been studied extensively, one of which is the so-called general VI (GVI) [17]. A general projection NN was presented in [18] for solving GVIs and related problems with many desirable properties such as global convergence, global asymptotic stability, and global exponential stability. In this paper, a modified version of the general projection $\mathrm{NN}$ will be presented for solving a special class of GVIs. It will be seen that more convergence and stability results can be obtained with many alternative conditions.

The remainder of this paper is organized as follows. The problem formulation and the proposed neural-network model are presented in the next section. Some preliminaries related to the problem are also presented in this section. In Section III, we extensively explore the capability of the proposed NN by studying its convergence and stability under various conditions. In Section IV, several special cases of GVIs and the corresponding NNs are discussed briefly. Illustrative examples are presented in Section V. Section VI gives the conclusions of this paper.

\section{PRELIMINARIES}

\section{A. Problem Formulation and Neural-Network Model}

Let $F: R^{n} \rightarrow R^{n}$ and $G: R^{n} \rightarrow R^{n}$ be continuous vectorvalued functions, and $\Omega$ be a closed convex set in $R^{n}$. Consider the following GVI [17]. Find $x^{*} \in R^{n}$ such that $G\left(x^{*}\right) \in \Omega$ and

$$
F\left(x^{*}\right)^{T}\left(x-G\left(x^{*}\right)\right) \geq 0, \quad \forall x \in \Omega .
$$

GVI includes many VIs as its special cases. For example, if $G(x)=x$ and $F(x)$ is an affine mapping, (1) reduces to the linear VI (LVI). Find $x^{*} \in \Omega$ such that

$$
\left(M x^{*}+p\right)^{T}\left(x-x^{*}\right) \geq 0, \quad \forall x \in \Omega
$$

where $M \in R^{n \times n}$ and $p \in R^{n}$. If $G(x)=x$ and $F(x)$ is not necessarily affine, $(1)$ reduces to the VI in the usual sense. Find $x^{*} \in \Omega$ such that

$$
F\left(x^{*}\right)^{T}\left(x-x^{*}\right) \geq 0, \quad \forall x \in \Omega .
$$

For another example, if both $G(x)$ and $F(x)$ are affine mappings, (1) becomes the generalized LVI (GLVI). Find $x^{*} \in R^{n}$ such that $N x^{*}+q \in \Omega$ and

$$
\left(M x^{*}+p\right)^{T}\left(x-N x^{*}-q\right) \geq 0, \quad \forall x \in \Omega
$$

where $M, N \in R^{n \times n}$ and $p, q \in R^{n}$. For solving (2) and (3), several NNs have been developed in [8]-[12] (for numerical algorithms one may refer to [6], [7] and references therein). 


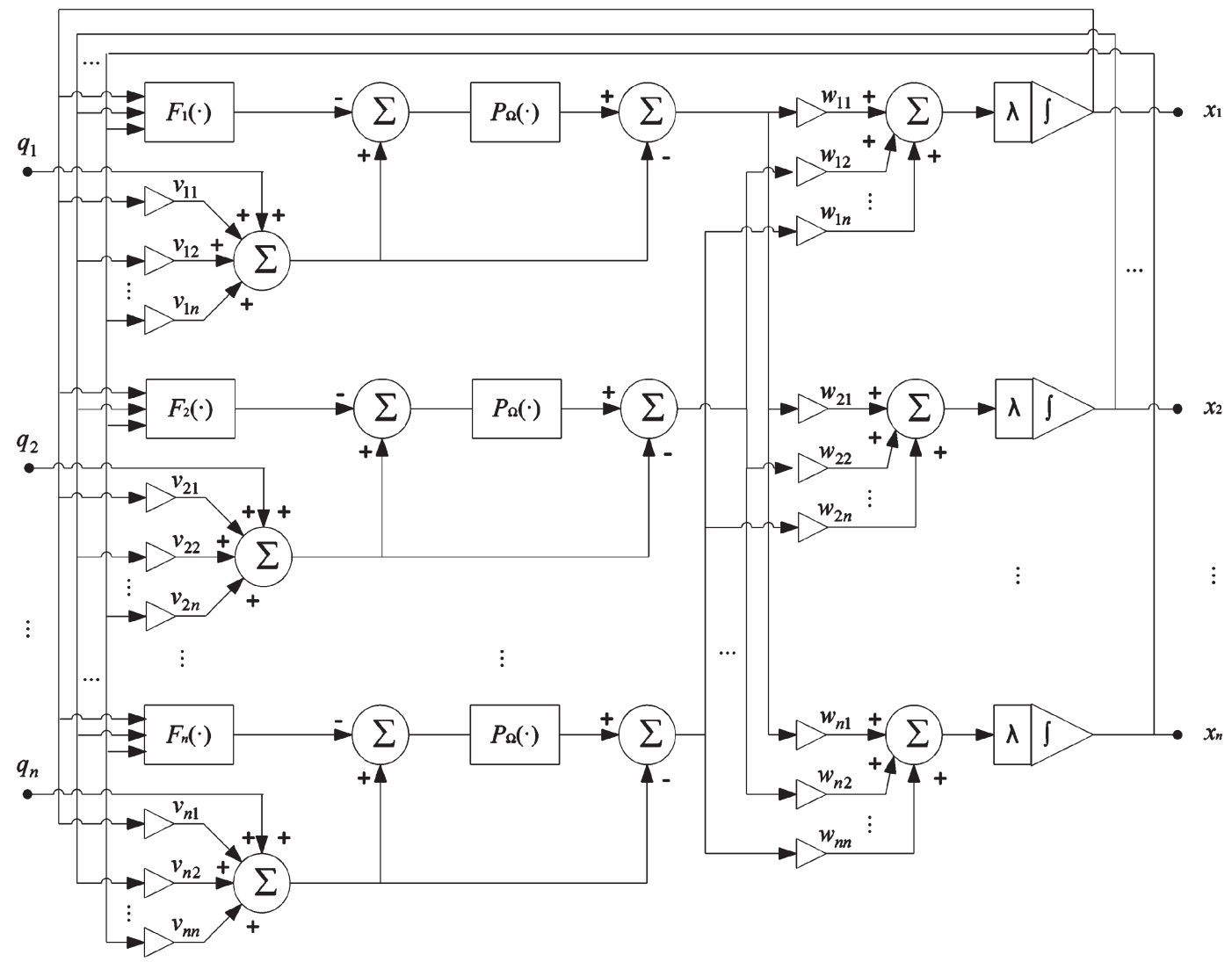

Fig. 1. Architecture of the NN in (8).

For solving (4), an NN can be found in [19]. For solving (1), a general projection NN (GPNN) with its dynamic system governed by

$$
\frac{d x}{d t}=\lambda\left\{-G(x)+P_{\Omega}(G(x)-F(x))\right\}
$$

is proposed, where $\lambda>0$ is a scaling factor, and $P_{\Omega}: R^{n} \rightarrow \Omega$ is a projection operator defined by

$$
P_{\Omega}(x)=\arg \min _{y \in \Omega}\|x-y\|
$$

where $\|\cdot\|$ denotes the $l_{2}$-norm of $R^{n}$.

In this paper, we consider another special case of GVIs in (1) by only requiring that $G(x)$ is affine. Find $x^{*} \in R^{n}$ such that $N x^{*}+q \in \Omega$ and

$$
F\left(x^{*}\right)^{T}\left(x-N x^{*}-q\right) \geq 0, \quad \forall x \in \Omega .
$$

Clearly, this problem also includes LVI in (2), VI in (3), and GLVI in (4) as special cases. A recurrent NN is proposed to solve (7) with its dynamical equation governed by

$$
\frac{d x}{d t}=\lambda W\left\{-N x+P_{\Omega}(N x+q-F(x))-q\right\}
$$

where $W$ is an $n \times n$ real matrix. Clearly, when $W=I$, the NN becomes a special case of the GPNN in (5). In this sense, the proposed $\mathrm{NN}$ in (8) can be regarded as a modified version of the GPNN for solving the GVI in (7).
In view of the fact that solving the GVI in (1) is equivalent to finding zeros of the generalized projection equation [20]

$$
P_{\Omega}(G(x)-F(x))-G(x)=0
$$

it is clear that the equilibria of the dynamic system (8) correspond to the solutions of (7) exactly if $W$ is nonsingular. Moreover, this dynamic system can be easily realized by a recurrent NN with a one-layer structure as shown in Fig. 1, where $W=\left\{w_{i j}\right\}, N=\left\{v_{i j}\right\}, q=\left\{q_{i}\right\}$, and $F(x)=\left\{F_{i}(x)\right\}$. We see from Fig. 1 that except for realizing the function $F(x)$, a circuit realizing of the NN consists of $4 n$ simple summators, $n$ integrators, $n$ units for computing the projection operator $P_{\Omega}(\cdot)$, and some weighted connections. In order to easily realize $P_{\Omega}(x)$, it must have an explicit expression for any $x \in R^{n}$. This is true in the following two cases. When $\Omega$ is a box set, i.e., $\Omega=\left\{x \in R^{n} \mid l_{i} \leq x_{i} \leq u_{i}, \forall i=1, \ldots, n\right\}$, then $P_{\Omega}(x)=\left[P_{\Omega}\left(x_{1}\right), \ldots, P_{\Omega}\left(x_{n}\right)\right]^{T}$ and

$$
P_{\Omega}\left(x_{i}\right)= \begin{cases}l_{i} & x_{i}<l_{i} \\ x_{i} & l_{i} \leq x_{i} \leq u_{i} \\ u_{i} & x_{i}>u_{i} .\end{cases}
$$

When $\Omega$ is a sphere set, i.e., $\Omega=\left\{x \in R^{n} \mid\|x-c\| \leq r\right.$, $r>0\}$, where $c \in R^{n}$ and $r \in R$ are two constants, then

$$
P_{\Omega}(x)= \begin{cases}x, & \|x-c\| \leq r \\ c+\frac{x-c}{\|x-c\|}, & \|x-c\|>r .\end{cases}
$$

Note that the GVI in (7) as well as in (1) does not always have a solution. For existence conditions for the solution of GVI, 
one may refer to [20]. Throughout this paper, we denoted the solution set of (7) by $\bar{\Omega}^{*}$, and assume $\bar{\Omega}^{*} \neq \emptyset$ and there exists a finite $x^{*} \in \bar{\Omega}^{*}$.

\section{B. Definitions and Lemmas}

Throughout this paper, the following notations are used. If $A$ is a symmetric matrix, then $\mu_{\min }(A)$ and $\mu_{\max }(A)$ denote the minimum and maximum eigenvalues of $A$, respectively, and $\|A\|=\sqrt{\mu_{\max }\left(A^{T} A\right)}$. If $x \in R^{n}$, then $\|x\|=\sqrt{\sum_{i=1}^{n} x_{i}^{2}}$. If $f(x)$ is a scalar-valued function, then $\nabla f(x) \in R^{n}$ stands for the gradient of $f(x)$. If $F(x)$ is a vector valued function mapping from $R^{n}$ to $R^{m}$, then $\nabla F(x) \in R^{m \times n}$ stands for the Jacobian matrix of $F(x)$. Define the following set closely related to the closed convex set $\Omega$ :

$$
\bar{\Omega}=\{x \mid N x+q \in \Omega\} .
$$

Clearly, $\bar{\Omega}$ is also a convex set. Moreover, $\bar{\Omega}^{*} \subset \bar{\Omega}$.

For the convenience of later discussion, it is necessary to introduce several definitions and lemmata.

Definition 1: Let $x(t)$ be a solution of a system $\dot{x}=F(x)$. The system is said to be globally convergent to a set $\hat{\Omega}$ if every solution of the system satisfies

$$
\lim _{t \rightarrow \infty} \operatorname{dist}(x(t), \hat{\Omega})=0
$$

where $\operatorname{dist}(x(t), \hat{\Omega})=\inf _{y \in \hat{\Omega}}\|x-y\|$.

Definition 2: A matrix function $M: R^{n} \rightarrow R^{n \times n}$ is said to be positive semidefinite on a set $\mathcal{K}$ if

$$
h^{T} M(x) h \geq 0, \quad \forall x \in \mathcal{K}, h \in R^{n} .
$$

$M(x)$ is positive definite on $\mathcal{K}$ if the above inequality holds wherever $h \neq 0$; it is uniformly positive definite on $\mathcal{K}$ if there exists a constant $\gamma>0$ such that

$$
h^{T} M(x) h \geq \gamma\|h\|^{2}, \quad \forall x \in \mathcal{K}, h \in R^{n} .
$$

Note that in the above definitions, we do not require that $M(x)$ is symmetric, which differs slightly from the corresponding definitions in the usual sense. An alternative way to understand the definitions is to interpret the positive semidefiniteness, positive definiteness, and uniform positive definiteness of an asymmetric matrix $M(x)$ as those of a symmetric matrix $M(x)+M(x)^{T}$ in the usual sense.

Definition 3: A mapping $F$ is said to be $G$-monotone on a set $\mathcal{K}$ if $\forall G(x), G(y) \in \mathcal{K}$

$$
(F(x)-F(y))^{T}(G(x)-G(y)) \geq 0 .
$$

Lemma 1: Assume that $F$ is continuously differentiable on an open convex set $\mathcal{K}$ and $G(x)=N x+q$ where $N \in R^{n \times n}$ and $q \in R^{n}$. Then, $F$ is $G$-monotone on $\mathcal{K}$ if and only if $\nabla F(x)^{T} N$ is positive semidefinite on $\overline{\mathcal{K}}$, where

$$
\overline{\mathcal{K}}=\left\{x \in R^{n} \mid N x+q \in \mathcal{K}\right\} .
$$

Proof: The $G$-monotonicity of $F$ on $\mathcal{K}$ implies that for any $N x+q \in \mathcal{K}$ and $N y+q \in \mathcal{K}$

$$
(F(x)-F(y))^{T}(N x+q-N y-q) \geq 0
$$

or

$$
\left(N^{T} F(x)-N^{T} F(y)\right)^{T}(x-y) \geq 0 .
$$

This is equivalent to say that the mapping $\tilde{F}(x)=N^{T} F(x)$ is monotone on $\overline{\mathcal{K}}$. Note that $\overline{\mathcal{K}}$ is also an open convex set. Then, the lemma follows from [7, Proposition 2.3.2, pp. 155-156].

Lemma 2 ([5, pp. 9-10]): Let $P_{\Omega}(\cdot)$ be defined by (6). For all $u \in R^{n}$ and all $v \in \Omega \subset R^{n}$, we have

$$
\left(P_{\Omega}(u)-u\right)^{T}\left(v-P_{\Omega}(u)\right) \geq 0
$$

and for all $u, v \in R^{n}$, we have

$$
\left\|P_{\Omega}(u)-P_{\Omega}(v)\right\| \leq\|u-v\|
$$

Lemma 3: Let a function $\phi: R^{n} \rightarrow R$ be defined as

$$
\phi(x)=\left\|N x+q-P_{\Omega}(N x+q)\right\|^{2}
$$

where $N$ and $q$ are parameters in (7). Then, $\phi(x)$ is continuously differentiable and its gradient is given by

$$
\nabla \phi(x)=2 N^{T}\left(N x+q-P_{\Omega}(N x+q)\right) .
$$

Proof: Define a function $\nu: R^{n} \times \Omega \rightarrow R$ by

$$
\nu(x, y) \triangleq\|N x+q-y\|^{2}
$$

which is clearly continuously differentiable. By the definition of the projection operator $P_{\Omega}$, we have

$$
\phi(x)=\min _{y \in \Omega} \nu(x, y) .
$$

The minimum on the right-hand side is uniquely attained at $y=P_{\Omega}(N x+q)$. It follows from [21, Ch. 4, Th. 1.7] (this theorem can be also found in [22, App.]) that $\phi(x)$ is differentiable and

$$
\nabla \phi(x)=\left.\nabla_{x} \nu(x, y)\right|_{y=P_{\Omega}(N x+q)} .
$$

This equation then gives (11).

Lemma 4: Define a function $V_{0}: R^{n} \rightarrow R$ by

$$
V_{0}(x)=-F(x)^{T}(H(x)-G(x))-\frac{1}{2}\|H(x)-G(x)\|^{2}
$$

where $H(x)=P_{\Omega}(G(x)-F(x))$. Then, $V_{0}(x) \geq 0$ for all $G(x) \in \Omega$ and $V_{0}(x)=0$ if and only if $x$ solves the GVI in (1). Moreover, if $F$ and $G$ are continuously differentiable, then $V_{0}$ is also continuously differentiable, and its gradient is given by

$$
\begin{aligned}
\nabla V_{0}(x)=\nabla G(x)^{T} F(x)+(\nabla G(x) & -\nabla F(x))^{T} \\
& \times(H(x)-G(x)) .
\end{aligned}
$$


Proof: Inspired by the work of Fukushima [23], we rewrite the function $V_{0}$ in the following equivalent form:

$$
V_{0}(x)=\frac{1}{2}\left\{\|F(x)\|^{2}-\|H(x)-(G(x)-F(x))\|^{2}\right\} .
$$

Consider the geometric meaning of the right-hand side. $\|F(x)\|$ is the distance from $G(x)-F(x)$ to $G(x)$, and $\| H(x)-$ $(G(x)-F(x)) \|$ is the distance from $G(x)-F(x)$ to its projection $H(x)$ onto $\Omega$. Hence, $V_{0}(x) \geq 0$ for all $G(x) \in \Omega$. Moreover, the definition of $H(x)$ implies that $V_{0}(x)=0$ if and only if $G(x)=H(x)$, which is equivalent to say that $x$ is a solution of the GVI in (1) [20]. Define a function $\nu: R^{n} \times \Omega \rightarrow$ $R$ as follows:

$$
\nu(x, y)=F(x)^{T}(y-G(x))+\frac{1}{2}\|y-G(x)\|^{2} .
$$

Because $F$ and $G$ are both continuously differentiable, so is $\nu(x, y)$. Consider the following constrained minimization problem:

$$
\min _{y \in \Omega} \nu(x, y) .
$$

It is equivalent to

$$
\min _{y \in \Omega}\|y-G(x)+F(x)\|^{2}
$$

the optimum of which is obviously uniquely determined as $y^{*}=H(x)$. Therefore, we have

$$
V_{0}(x)=-\min _{y \in \Omega} \nu(x, y)
$$

It follows from [21, Ch. 4, Th. 1.7] that $V_{0}(x)$ is differentiable and

$$
\nabla V_{0}(x)=-\left.\nabla_{x} \nu(x, y)\right|_{y=H(x)} .
$$

This equation then gives (13).

Lemma 5: Assume that $F(x)$ is locally Lipschitz continuous in $R^{n}$ and $W$ in (8) is not equal to zero. For any $x_{0} \in R^{n}$, there exists a unique solution $x(t)$ for (8) with $x\left(t_{0}\right)=x_{0}$ over $\left[t_{0}, T\right)$, where $T \geq t_{0}$.

The proof of Lemma 5 parallels that of [18, Lemma 2], and is thus omitted. In what follows, we assume that $F(x)$ is continuously differentiable so that $F(x)$ is locally Lipschitz continuous.

\section{Stability ANALYSis}

The stability analysis of the proposed NN will be conducted based on the Lyapunov theorem and LaSalle's invariant set theorem [24]. The application of these theorems to recurrent NNs has a profound background and may be traced back to the analysis of nonlinear difference-differential equations in learning theory or prediction theory [25]-[27], and then to the analysis of Hopfield NNs [28]. Some recent applications can be found in, to list a few, [12]-[16], [29]-[32], and references therein.

\section{A. Nonsingular Matrix $N$}

We first consider solving the GVI in (7) with a positive definite matrix $N$ by using the NN in (8) with $W=I$. Then, the NN becomes exactly the GPNN in (8) proposed in [18], where several stability results about the GPNN with general $G(x)$ are presented. Based on this special case of $G(x)$, (i.e., an affine function,) the following results can be obtained.

Theorem 1: Suppose that the function $U(x)=N x+q-$ $F(x)$ is globally Lipschitz continuous with constant $L$, i.e.,

$$
\|U(x)-U(y)\| \leq L\|x-y\|, \quad \forall x, y \in R^{n} .
$$

If $N$ is positive definite and $\mu_{\min }\left(N^{S}\right)>L$, where $N^{S}=$ $\left(N+N^{T}\right) / 2$, then the NN in (8) with $W=I$ is exponentially stable at the unique solution of the GVI in (7).

Proof: Let $x^{*}$ be a finite solution of the GVI in (7). Define the Lyapunov function

$$
V(x(t))=\frac{1}{2 \lambda}\left\|x(t)-x^{*}\right\|^{2}, \quad \forall t \geq t_{0} .
$$

Compute the time derivative of $V(x(t))$ along the trajectory of (8) with $W=I$

$$
\begin{aligned}
\frac{d V}{d t}= & \left(x-x^{*}\right)^{T}\left[-N x-q+P_{\Omega}(N x+q-F(x))\right] \\
= & \left(x-x^{*}\right)^{T}\left[-N x+N x^{*}+P_{\Omega}(U(x))-P_{\Omega}\left(U\left(x^{*}\right)\right)\right] \\
= & -\left(x-x^{*}\right)^{T} N\left(x-x^{*}\right)+\left(x-x^{*}\right)^{T} \\
& \times\left[P_{\Omega}(U(x))-P_{\Omega}\left(U\left(x^{*}\right)\right)\right] \\
\leq & -\mu_{\min }\left(N^{S}\right)\left\|x-x^{*}\right\|^{2}+\left\|x-x^{*}\right\|\left\|U(x)-U\left(x^{*}\right)\right\| \\
\leq & -\beta\left\|x-x^{*}\right\|^{2}=-2 \lambda \beta V(x(t)), \quad \forall t \geq t_{0}
\end{aligned}
$$

where

$$
\beta=\mu_{\min }\left(N^{S}\right)-L>0 .
$$

In the above reasoning, Lemma 2 is used. It follows:

$$
V(x(t)) \leq V\left(x\left(t_{0}\right)\right) \exp \left(-2 \lambda \beta\left(t-t_{0}\right)\right)
$$

and

$$
\left\|x(t)-x^{*}\right\| \leq\left\|x(t)-x^{*}\right\| \exp \left(-\lambda \beta\left(t-t_{0}\right)\right), \quad \forall t \geq t_{0} .
$$

Hence, the NN is exponentially stable at $x^{*}$.

In Theorem 1, the matrix $N$ is required to be nonsingular. In fact, if this is true, we can choose $W=N^{-1}$ in (8) and obtain more results for the NN. Define $y=N x+q$, and then (8) with $W=N^{-1}$ can be converted into

$$
\frac{d y}{d t}=\lambda\left\{-y+P_{\Omega}\left(y-F\left(N^{-1} y-N^{-1} q\right)\right)\right\}
$$

which is a projection $\mathrm{NN}$ discussed extensively in the literature (e.g., [11], [12], and [18]), and all stability results therein can be applied to this NN. For example, some recent stability results for the projection $\mathrm{NN}$ are combined into Theorem 2 . 
Theorem 2: Suppose that $N$ is nonsingular. Consider the $\mathrm{NN}$ in (8) with $W=N^{-1}$ and $x\left(t_{0}\right) \in \bar{\Omega}$ for solving the GVI in (7).

1) The $\mathrm{NN}$ is stable in the sense of Lyapunov and globally converges to the solution set of GVI if $\nabla F(x)^{T} N$ is symmetric and $\Omega$ is bounded.

2) The NN is stable in the sense of Lyapunov and globally converges to an exact solution of GVI if either of the following conditions is satisfied.

a) $\nabla F(x)^{T} N$ is symmetric and positive definite on $\bar{\Omega}$.

b) $\nabla F(x)^{T} N$ is uniformly positive definite on $\bar{\Omega}$.

3) The NN is exponentially convergent to the unique solution of GVI if either of the following conditions is satisfied.

a) $\nabla F(x)^{T} N$ is symmetric and positive definite on $\bar{\Omega}$.

b) $\nabla F(x)^{T} N$ is uniformly positive definite on $\bar{\Omega}$.

Proof: Consider the equivalent form (14) of the $\mathrm{NN}$ in (8). We have $\nabla F\left(N^{-1} y-N^{-1} q\right)=\nabla F(x) N^{-1}$. Note that the positive (semi)definiteness of $\nabla F(x)^{T} N$ implies the positive (semi)definiteness of $\nabla F(x) N^{-1}$, and that the uniform positive definiteness of $\nabla F(x)^{T} N$ implies the uniform positive definiteness of $\nabla F(x) N^{-1}$. Then, part 1) follows from [11, Th. 1]; part 2) follows from [12, Th. 2.1] and [11, Th. 3]; and part 3) follows from [12, Th. 2.2] and [18, Th. 4].

\section{B. Singular Matrix $N$}

When the matrix $N \in R^{n \times n}$ in (7) is singular, it is also possible to solve the problem with the NN in (8) by choosing appropriate $W$. Without loss of generality, we assume that $\operatorname{Rank}(N)=m<n$. According to the matrix theory, there always exist orthogonal matrices $P$ and $Q$ such that

$$
N=P \Lambda Q^{T}
$$

where $\Lambda=\operatorname{diag}\left(\sigma_{1}, \ldots, \sigma_{m}, 0, \ldots, 0\right)$ and $\sigma_{1}, \ldots, \sigma_{m}$ are the singular values of $N$ which are all positive. Choose

$$
\tilde{N}=P A Q^{T}
$$

where $A=\operatorname{diag}\left(\sigma_{1}, \ldots, \sigma_{m}, 1, \ldots, 1\right)$, and consider the $\mathrm{NN}$ in (8) with $W=\tilde{N}^{-1}=Q A^{-1} P^{T}$. Clearly, if $m=n, \tilde{N}=N$. In other words, the NN with $W=\tilde{N}^{-1}$ is a generalization of the NN with $W=N^{-1}$ discussed in Section III-A. We have the following results.

Theorem 3: If $\nabla F(x)^{T} N$ is positive semidefinite on $R^{n}$ and $\nabla F(x)^{T} \tilde{N}$ is symmetric and positive definite on $R^{n}$, then the $\mathrm{NN}$ in (8) with $W=\tilde{N}^{-1}$ is stable in the sense of Lyapunov and globally convergent to a point in $\bar{\Omega}^{*}$. In particular, if (7) has a unique solution $x^{*}$, the $\mathrm{NN}$ is globally asymptotically stable at $x^{*}$.

Proof: Clearly $\tilde{N}^{-1} \neq 0$. By Lemma 5 , there exists a unique continuous solution $x(t)$ for system (8) over $\left[t_{0}, T\right)$. Let $U(x) \triangleq \tilde{N}^{T} F(x)$ and $x^{*}$ be a finite point in $\bar{\Omega}^{*}$. Consider the following function:

$$
V(x)=V_{1}(x)+\frac{1}{2 \lambda}\left\|\Lambda Q^{T}\left(x-x^{*}\right)\right\|^{2}
$$

where

$$
V_{1}(x)=\frac{1}{\lambda} \int_{0}^{1}\left(x-x^{*}\right)^{T}\left[U\left(x^{*}+t\left(x-x^{*}\right)\right)-U\left(x^{*}\right)\right] d t
$$

and $\Lambda, Q$ are defined as same as in (15). Since $\nabla U(x)=$ $\tilde{N}^{T} \nabla F(x)$ is symmetric and positive definite on $R^{n}, V_{1}(x)$ is continuously differentiable and strictly convex on $R^{n}$ and $\nabla V_{1}(x)=\lambda^{-1}\left(U(x)-U\left(x^{*}\right)\right)$ [6]. Then, $x^{*}$ corresponds to the unique minimum of $V_{1}$. The definition of $V_{1}$ implies that $V_{1}\left(x^{*}\right)=0$. As $x^{*}$ is finite, the function $V_{1}(x)$, and accordingly $V(x)$, is radially unbounded. Moreover, since $x^{*} \in \bar{\Omega}^{*}$, by (7), we have

$$
F\left(x^{*}\right)^{T}\left(P_{\Omega}(N x+q-F(x))-N x^{*}-q\right) \geq 0 .
$$

In Lemma 2, let $u=N x+q-F(x)$ and $v=N x^{*}+q$, we have

$$
\begin{aligned}
& {\left[P_{\Omega}(N x+q-F(x))-N x-q+F(x)\right]^{T} } \\
& \cdot\left[N x^{*}+q-P_{\Omega}(N x+q-F(x))\right] \geq 0 .
\end{aligned}
$$

Summing the two resulting inequalities above yields

$$
\begin{aligned}
& {\left[F(x)-F\left(x^{*}\right)+N x-N x^{*}\right]^{T}} \\
& \left.\quad \cdot P_{\Omega}(N x+q-F(x))-N x-q\right] \\
& \quad \leq-\left(N x-N x^{*}\right)^{T}\left(F(x)-F\left(x^{*}\right)\right) \\
& \quad-\left\|N x+q-P_{\Omega}(N x+q-F(x))\right\|^{2} .
\end{aligned}
$$

By noticing that $\tilde{N}^{-1}=Q A^{-1} P^{T}$ and $\tilde{A}^{-1} \Lambda^{2}=\Lambda$, we can deduce that for $x \in \bar{\Omega}$

$$
\begin{aligned}
\frac{d V(x)}{d t}= & \nabla V(x)^{T} \frac{d x(t)}{d t} \\
= & {\left[F(x)-F\left(x^{*}\right)\right]^{T}\left[-N x-q+P_{\Omega}(N x+q-F(x))\right] } \\
& +\left[\Lambda Q^{T}\left(x-x^{*}\right)\right]^{T} \Lambda Q^{T} \\
& \cdot \tilde{N}^{-1}\left[-N x-q+P_{\Omega}(N x+q-F(x))\right] \\
= & {\left[F(x)-F\left(x^{*}\right)\right]^{T}\left[-N x-q+P_{\Omega}(N x+q-F(x))\right] } \\
& +\left[P A^{-1} \Lambda^{2} Q^{T}\left(x-x^{*}\right)\right]^{T} \\
& \cdot\left[-N x-q+P_{\Omega}(N x+q-F(x))\right] \\
= & {\left[F(x)-F\left(x^{*}\right)+N x-N x^{*}\right]^{T} } \\
& \cdot\left[-N x-q+P_{\Omega}(N x+q-F(x))\right] \\
\leq & -\left(N x-N x^{*}\right)^{T}\left(F(x)-F\left(x^{*}\right)\right) \\
& -\left\|N x+q-P_{\Omega}(N x+q-F(x))\right\|^{2} .
\end{aligned}
$$


Since $\nabla F(x)^{T} N$ is positive semidefinite on $R^{n}$, by Lemma 1, $F(x)$ is $G$-monotone on $R^{n}$ with respect to $G(x)=N x+q$. Then

$$
\frac{d V(x)}{d t} \leq-\left\|N x+q-P_{\Omega}(N x+q-F(x))\right\|^{2} \leq 0 .
$$

Therefore, $V(x)$ is a Lyapunov function and the $\mathrm{NN}$ in (8) is stable in the Lyapunov sense. Clearly, $d V(x) / d t=0$ if and only if $x \in \bar{\Omega}^{*}$. Then

$$
\left\{x(t) \mid t_{0} \leq t<T\right\} \subset \mathcal{S}=\left\{x \in \bar{\Omega} \mid V(x) \leq V\left(x\left(t_{0}\right)\right)\right\} .
$$

As $V(x)$ is radially unbounded, $\mathcal{S}$ is bounded. On one hand, this fact implies that $T=+\infty$ and that the NN converges to $\bar{\Omega}^{*}$ according to the LaSalle's invariant set theorem [24]. On the other hand, It implies that there exists a convergent subsequence $\left\{x\left(t_{k}\right)\right\}$ such that

$$
\lim _{k \rightarrow \infty} x\left(t_{k}\right)=\hat{x}
$$

where $\hat{x} \in \bar{\Omega}^{*}$. Finally, define another Lyapunov function

$$
\begin{aligned}
\hat{V}(x)=\frac{1}{\lambda} \int_{0}^{1}(x-\hat{x})^{T}[U(\hat{x}+t(x-\hat{x}))-U(\hat{x})] d t & \\
& +\frac{1}{2 \lambda}\left\|\Lambda Q^{T}(x-\hat{x})\right\|^{2} .
\end{aligned}
$$

It is easy to see that $\hat{V}(x)$ decreases along the trajectory of (8) and satisfies $\hat{V}(\hat{x})=0$. Therefore, for any $\varepsilon>0$, there exists $q>0$ such that, for all $t \geq t_{q}$

$$
\hat{V}(x(t)) \leq \hat{V}\left(x\left(t_{q}\right)\right)<\varepsilon .
$$

As $\hat{V}(x)$ is strictly convex with its minimum $\hat{V}(\hat{x})=0$, we have $\lim _{t \rightarrow \infty} x(t)=\hat{x}$. Therefore, the $\mathrm{NN}$ in (8) with $W=$ $\tilde{N}^{-1}$ is globally convergent to a solution of (7). In particular, if (7) has a unique solution $x^{*}$, the $\mathrm{NN}$ is globally asymptotically stable at $x^{*}$.

\section{Block Diagonal Matrix $N$}

Now, we consider solving the GVI in (7) with $N \in R^{n \times n}$ being in the following form:

$$
N=\left(\begin{array}{cc}
N_{I} & 0 \\
0 & 0
\end{array}\right)
$$

where $N_{I} \in R^{m}$ is nonsingular and $m<n$. The problem can be solved by using the NN designed in Section III-B. Moreover, because of the special structure of $N$, the matrix $\tilde{N}$ in (16) has a quite simple form. By singular value decomposition, $N_{I}$ can be factorized as $N_{I}=P_{I} A_{I} Q_{I}^{T}$, where $P_{I}, Q_{I}$ are two orthogonal matrices and $A_{I}$ is a diagonal matrix with its entries being the singular values of $N_{I}$ that are all positive. Select

$$
P=\left(\begin{array}{cc}
P_{I} & 0 \\
0 & I
\end{array}\right) \quad Q=\left(\begin{array}{cc}
Q_{I} & 0 \\
0 & I
\end{array}\right) \quad \Lambda=\left(\begin{array}{cc}
A_{I} & 0 \\
0 & 0
\end{array}\right) .
$$

It is verified that $P$ and $Q$ are the orthogonal matrices and that $N=P \Lambda Q^{T}$. Therefore

$$
\tilde{N}=P\left(\begin{array}{cc}
A_{I} & 0 \\
0 & I
\end{array}\right) \quad Q^{T}=\left(\begin{array}{cc}
N_{I} & 0 \\
0 & I
\end{array}\right) .
$$

If $\Omega$ is a box set, then it can be partitioned as $\Omega=\Omega_{I} \times \Omega_{I I}$, where $\Omega_{I} \subset R^{m}$ and $\Omega_{I I} \subset R^{n-m}$ are also two boxes, and the $\mathrm{NN}$ in (8) with $W=\tilde{N}^{-1}$ can be partitioned as follows:

$$
\left\{\begin{array}{l}
\frac{d x_{I}}{d t}=\lambda N_{I}^{-1}\left\{-N_{I} x_{I}+P_{\Omega_{I}}\left(N_{I} x_{I}+q_{I}-F_{I}(x)\right)-q_{I}\right\} \\
\frac{d x_{I I}}{d t}=\lambda\left\{P_{\Omega_{I I}}\left(q_{I I}-F_{I I}(x)\right)-q_{I I}\right\}
\end{array}\right.
$$

where the meanings of the parameters with subscripts $I$ and $I I$ such as $x_{I}, x_{I I}$ are self-evident. The second part of the $\mathrm{NN}$ indicates that $q_{I I}$ must reside in $\Omega_{I I}$; otherwise, the $\mathrm{NN}$ would have no equilibrium point, and correspondingly $\bar{\Omega}^{*}=\emptyset$. Hence, when we talk about this NN, this condition is always assumed true.

Some conditions have been presented in Theorem 3 for ascertaining the global convergence of the $\mathrm{NN}$ in (8) with $W=\tilde{N}^{-1}$. In this section, we seek more results for this NN based on the special structure of $N$ defined in (17).

The following theorem claims that $\bar{\Omega}$ is actually an invariant and asymptotically attractive set for the NN in (8) with $W=$ $\tilde{N}^{-1}$. The proof is in the spirit of [10, Th. 3.2] and makes use of Lemma 3.

Theorem 4: Assume that $\Omega$ is a box set and that $N$ is a block diagonal matrix defined in (17). Then, the trajectory $x(t)$ of the NN in (8) with $W=\tilde{N}^{-1}$ will approach $\bar{\Omega}$ exponentially when $x\left(t_{0}\right) \notin \bar{\Omega}$ and stay inside $\bar{\Omega}$ when $x\left(t_{0}\right) \in \bar{\Omega}$ for all $t \geq t_{0}$.

Proof: Since $\tilde{N}^{-1} \neq 0$, by Lemma 5 , there is a unique solution $x(t)$ of (8) over $\left[t_{0}, T\right)$ for any initial point $x\left(t_{0}\right) \in$ $R^{n}$. When $x\left(t_{0}\right) \notin \bar{\Omega}$, i.e., $N x\left(t_{0}\right)+q \notin \Omega$, without loss of generality, we assume that

$$
N x(t)+q \notin \Omega, \quad \forall t \geq t_{0} .
$$

Let a function $\phi(x(t))$ be defined in Lemma 3. Then

$$
\begin{aligned}
\frac{d \phi(x(t))}{d t}= & \nabla \phi(x)^{T} \frac{d x(t)}{d t} \\
= & 2\left[N x+q-P_{\Omega}(N x+q)\right]^{T} N \\
& \cdot \lambda \tilde{N}^{-1}\left[-N x-q+P_{\Omega}(N x+q-F(x))\right] \\
= & 2 \lambda\left[N_{I} x_{I}+q_{I}-P_{\Omega_{I}}\left(N_{I} x_{I}+q_{I}\right)\right]^{T} \\
& \cdot\left[P_{\Omega_{I}}\left(N_{I} x_{I}+q_{I}-F_{I}(x)\right)-P_{\Omega_{I}}\left(N_{I} x_{I}+q_{I}\right)\right] \\
& -2 \lambda\left\|N_{I} x_{I}+q_{I}-P_{\Omega_{I}}\left(N_{I} x_{I}+q_{I}\right)\right\|^{2} .
\end{aligned}
$$


In Lemma 2, let $u=N_{I} x_{I}+q_{I}, v=P_{\Omega_{I}}\left(N_{I} x_{I}+q_{I}-\right.$ $\left.F_{I}(x)\right)$, then

$$
\begin{aligned}
{\left[N_{I} x_{I}+q_{I}-P_{\Omega_{I}}\left(N_{I} x_{I}+q_{I}\right)\right]^{T} } & \\
\cdot & {\left[P_{\Omega_{I}}\left(N_{I} x_{I}+q_{I}-F_{I}(x)\right)-P_{\Omega_{I}}\left(N_{I} x_{I}+q_{I}\right)\right] \leq 0 }
\end{aligned}
$$

which implies

$$
\begin{aligned}
d \phi(t) / d t & \leq-2 \lambda\left\|N_{I} x_{I}+q_{I}-P_{\Omega_{I}}\left(N_{I} x_{I}+q_{I}\right)\right\|^{2} \\
& =-2 \lambda \phi(t)
\end{aligned}
$$

in view of the fact $q_{I I} \in \Omega_{I I}$. Hence, $\forall t \in\left[t_{0}, T\right)$

$$
\begin{aligned}
\| N x(t)+q- & P_{\Omega}(N x(t)+q) \| \\
& \leq\left\|N x\left(t_{0}\right)+q-P_{\Omega}\left(N x\left(t_{0}\right)+q\right)\right\| e^{-\lambda\left(t-t_{0}\right)} .
\end{aligned}
$$

Therefore, the trajectory of $N x(t)+q$ will approach $\Omega$ exponentially, and accordingly the trajectory of $x(t)$ will approach $\bar{\Omega}$ exponentially. We now prove the second part of the theorem. Suppose when $N x\left(t_{0}\right)+q \in \Omega$, there is $t_{2}>t_{1}$ such that $N x(t)+q \in \Omega$ for $t \in\left[t_{0}, t_{1}\right]$ and $N x(t)+q \notin \Omega$ for $t \in$ $\left(t_{1}, t_{2}\right]$, then $\phi\left(t_{1}\right)=0, \phi\left(t_{2}\right)>0$. We show this could not happen by contradiction. By the mean value theorem, there exists $\hat{t} \in\left(t_{1}, t_{2}\right)$ such that

$$
\frac{d \phi(\hat{t})}{d t}=\frac{\phi\left(t_{2}\right)-\phi\left(t_{1}\right)}{t_{2}-t_{1}}>0 .
$$

But, we have obtained above that $d \phi(t) / d t \leq 0$ for all $t \geq t_{0}$, which is a contradiction. Thus, $N x(t)+q \in \Omega$ over $\left[t_{0}, T\right)$ if $N x\left(t_{0}\right)+q \in \Omega$. In other words, $x(t) \in \bar{\Omega}$ over $\left[t_{0}, T\right)$ if $x\left(t_{0}\right) \in \bar{\Omega}$. The proof is complete.

To guarantee the global stability of the NN with $W=\tilde{N}^{-1}$, Theorem 3 requires that $\nabla F(x)^{T} \tilde{N}$ is symmetric and positive definite. In the case when $N$ is a block diagonal matrix, we will show that the symmetry of $\nabla F(x)^{T} \tilde{N}$ can be replaced by some other conditions without affecting the stability properties of the NN. In establishing the results, we will use the function $V_{0}$ defined in Lemma 4 to construct a Lyapunov function for the stability analysis of the NN. Lemma 4 has revealed many desired properties of this function. However, it would be more desirable if the function is radially unbounded, i.e., $V_{0}(x) \rightarrow \infty$, $\forall\|x\| \rightarrow \infty$. Additionally, in many cases, this is true. For example, let $F(x)=2 x, G(x)=0$, where $x \in R$, then $V_{0}(x)=$ $2 x^{2}-\left(P_{\Omega}(-2 x)+2 x\right)^{2} / 2$. If $\Omega=[-1, \infty)$, then $V_{0} \rightarrow \infty$, $\forall|x| \rightarrow \infty$. But in some cases, it is not true. Moreover, it is difficult to characterize this property for the function. What we know at present is that this property depends not only on $F$ and $G$ but also on $\Omega$. Consider the same example above and let $\Omega=[0, \infty)$. It is easy to see that $V_{0}(x)=0$ when $x \geq 0$. Thus, $V_{0}$ is not radially unbounded in this case. Further studies in this regard are needed. Anyway, if this property of $V_{0}$ holds, we have the following results.

Theorem 5: Assume that $\Omega$ is a box and that $N$ is a block diagonal matrix defined in (17). If $V_{0}$ defined in (12) is radially unbounded, $\nabla F(x)^{T} N$ is positive semidefinite on $\bar{\Omega}$, and $\nabla F(x)^{T} \tilde{N}$ is positive definite on $\bar{\Omega}$, then the $\mathrm{NN}$ in (8) with $W=\tilde{N}^{-1}$ and $x\left(t_{0}\right) \in \bar{\Omega}$ is stable in the sense of Lyapunov and globally convergent to $\bar{\Omega}^{*}$. In particular, if $\bar{\Omega}^{*}$ has a unique point $x^{*}$, the $\mathrm{NN}$ is globally asymptotically stable at $x^{*}$.

Proof: By Theorem 4, $N x(t)+q \in \Omega$ for $t \geq t_{0}$ as $x\left(t_{0}\right) \in \bar{\Omega}$. Consider the following function:

$$
V(x)=\frac{1}{\lambda}\left[V_{0}(x)+\frac{1}{2}\left\|N x-N x^{*}\right\|^{2}\right], \quad \forall N x\left(t_{0}\right)+q \in \Omega
$$

where $V_{0}(x)$ is defined by (12) with $G(x)=N x+q$ and $x^{*}$ is a finite point in $\bar{\Omega}^{*}$. According to Lemma $4, V(x) \geq 0$ for all $x$ satisfying $N x+q \in \Omega$ and $V(x)=0$ if and only if $x=x^{*}$. In Lemma 2 , by letting $u=N_{I} x_{I}+q_{I}-F_{I}(x)$ and $v=N_{I} x_{I}^{*}+$ $q_{I}$, we have

$$
\left(H_{I}(x)-N_{I} x_{I}-q_{I}+F_{I}(x)\right)^{T}\left(N_{I} x_{I}^{*}+q_{I}-H_{I}(x)\right) \geq 0
$$

where $H_{I}(x)=P_{\Omega_{I}}\left(N_{I} x_{I}+q_{I}-F_{I}(x)\right)$, and

$$
\begin{aligned}
&\left(H_{I}(x)-N_{I} x_{I}-q_{I}+F_{I}(x)\right)^{T} \\
& \cdot\left(N_{I} x_{I}^{*}-N_{I} x_{I}+N_{I} x_{I}+q_{I}-H_{I}(x)\right) \geq 0
\end{aligned}
$$

which follows

$$
\begin{aligned}
& \left(F_{I}(x)+N_{I} x_{I}-N_{I} x_{I}^{*}\right)^{T}\left(H_{I}(x)-N_{I} x_{I}-q_{I}\right) \\
& +\left\|H_{I}(x)-N_{I} x_{I}-q_{I}\right\|^{2} \\
& \quad \leq-F_{I}(x)^{T}\left(N_{I} x_{I}-N_{I} x_{I}^{*}\right) \\
& \quad=-F(x)^{T}\left(N x-N x^{*}\right) .
\end{aligned}
$$

Let $H(x)=P_{\Omega}(N x+q-F(x))$. By using Lemma 4 and the above inequality, we deduce

$$
\begin{aligned}
\frac{d V}{d t}= & \left(\frac{d V}{d x}\right)^{T} \frac{d x}{d t} \\
= & \lambda^{-1}\left[N^{T} F(x)+(N-\nabla F(x))^{T}(H(x)-N x-q)\right. \\
& \left.\quad+N^{T}\left(N x-N x^{*}\right)\right]^{T}(d x / d t) \\
= & \left(F(x)+N x-N x^{*}\right)^{T} N \tilde{N}^{-1}(H(x)-N x-q) \\
& +(H(x)-N x-q)^{T} N \tilde{N}^{-1}(H(x)-N x-q) \\
& -(H(x)-N x-q)^{T} \nabla F(x) \tilde{N}^{-1}(H(x)-N x-q) \\
= & \left(F_{I}(x)+N_{I} x_{I}-N_{I} x_{I}^{*}\right)^{T}\left(H_{I}(x)-N_{I} x_{I}-q_{I}\right) \\
& +\left\|H_{I}(x)-N_{I} x_{I}-q_{I}\right\|^{2} \\
& -(H(x)-N x-q)^{T} \nabla F(x) \tilde{N}^{-1}(H(x)-N x-q) \\
\leq & -F(x)^{T}\left(N x-N x^{*}\right) \\
& -(H(x)-N x-q)^{T} \nabla F(x) \tilde{N}^{-1}(H(x)-N x-q) .
\end{aligned}
$$

On one side, the positive semidefiniteness of $\nabla F(x)^{T} N$ on $\bar{\Omega}$ implies that $F(x)$ is $G$-monotone on $\Omega$, which means

$$
\left(F(x)-F\left(x^{*}\right)\right)^{T}\left(N x-N x^{*}\right) \geq 0, \quad \forall x \in \bar{\Omega} .
$$


Together with (7), this inequality implies

$$
F(x)^{T}\left(N x-N x^{*}\right) \geq 0, \quad \forall x \in \bar{\Omega} .
$$

On the other side, the positive definiteness of $\nabla F(x)^{T} \tilde{N}$ on $\bar{\Omega}$ implies the positive definiteness of $\nabla F(x) \tilde{N}^{-1}$ on $\bar{\Omega}$. Thus

$$
(H(x)-N x-q)^{T} \nabla F(x) \tilde{N}^{-1}(H(x)-N x-q) \geq 0
$$

for all $x \in \bar{\Omega}$ and the equality holds if and only if $H(x)-$ $N x-q=0$, or $d x / d t=0$. Therefore, $V(x)$ is a Lyapunov function and the $\mathrm{NN}$ in (8) is stable in the Lyapunov sense. As $V_{0}(x)$ is radially unbounded, so is $V(x)$, and the following set:

$$
\mathcal{S}=\left\{x \in \bar{\Omega} \mid V(x) \leq V\left(x\left(t_{0}\right)\right)\right\}
$$

is bounded. According to the LaSalle's invariant set theorem [24], the NN will converge to the largest invariant set in $\mathcal{S}$, that is, $\bar{\Omega}^{*}$. In particular, if (7) has a unique solution $x^{*}$, the $\mathrm{NN}$ is globally asymptotically stable at $x^{*}$.

Remark 1: In Section III-C, the GVI with $N$ being in the form of (17) is considered only. We remark that if there are multiple blocks in the diagonal of $N$, i.e.,

$$
N=\left(\begin{array}{ccccc}
N_{I} & & & & \\
& 0 & & & \\
& & N_{I I} & & \\
& & & \ddots & \\
& & & & 0
\end{array}\right)
$$

where $N_{I}, N_{I I}, \ldots$ are nonsingular, the $\mathrm{NN}$ in (8) with $W=$ $\tilde{N}^{-1}$ can also be used to solve the problem, where $\tilde{N}$ is chosen as follows: 1) let $\tilde{N}=N$ and 2) change all zeros in the diagonal to ones except those in the $N_{I}, N_{I I}, \ldots$ blocks. Then, all the stability results discussed in Section III-C are still valid for the NN.

\section{Special Cases}

Consider the following generalized nonlinear complementarity problem (GNCP). Find $x^{*} \in R^{n}$ such that

$$
N x^{*}+q \geq 0 \quad F\left(x^{*}\right) \geq 0 \quad\left(N x^{*}+q\right)^{T} F\left(x^{*}\right)=0
$$

where $N \in R^{n \times n}$, and $F(x)$ is a differentiable vector-valued function from $R^{n}$ into $R^{n}$. If $N=I$ and $q=0$, the above GNCP reduces to an NCP in the usual sense, which has been studied extensively (see, e.g., [6], [7], and references therein). We show that the GNCP defined in (19) is equivalent to the GVI in (7) with $\Omega=R_{+}^{n}$, i.e., the nonnegative quadrant of $R^{n}$. On one hand, if $x^{*}$ is a solution to the GNCP, then

$$
\begin{aligned}
F\left(x^{*}\right)^{T}\left(x-N x^{*}-q\right) & =F\left(x^{*}\right)^{T} x-F\left(x^{*}\right)^{T}\left(N x^{*}+q\right) \\
& =F\left(x^{*}\right)^{T} x \geq 0, \quad \forall x \in R_{+}^{n} .
\end{aligned}
$$

On the other hand, assume $x^{*}$ is a solution to the GVI in (7). Then, $N x^{*}+q \geq 0$. Substituting $x=0$ into (7) gives $F\left(x^{*}\right)^{T}\left(N x^{*}+q\right) \leq 0$. Let $x=2\left(N x^{*}+q\right)$, then $x \geq 0$. Substituting $x$ into (7) gives $F\left(x^{*}\right)^{T}\left(N x^{*}+q\right) \geq 0$. Thus, $F\left(x^{*}\right)^{T}\left(N x^{*}+q\right)=0$. In turn, this yields $F\left(x^{*}\right)^{T} x \geq 0$ for all $x \in R_{+}^{n}$; thus $F\left(x^{*}\right) \geq 0$. Therefore, $x^{*}$ is also a solution to the GNCP.

Because of the equivalence between GVI and GNCP, the proposed NN in (8) can be used to solve GNCP in (19) as well. Specifically, the NN for this purpose can be described as follows:

$$
\frac{d x}{d t}=\lambda W\left\{-N x+(N x+q-F(x))^{+}-q\right\}
$$

where $(x)^{+}=\left[\left(x_{1}\right)^{+}, \ldots,\left(x_{n}\right)^{+}\right]$and $\left(x_{i}\right)^{+}=\max \left(x_{i}, 0\right)$.

In the following, we consider the linear case of GVI and GNCP. If $F(x)$ is an affine mapping in (7), i.e., $F(x)=$ $M x+p$, where $M \in R^{n \times n}$ and $q \in R^{n}$. Then, (7) becomes exactly (4), which represents the GLVI studied in [33]. To solve the problem, the $\mathrm{NN}$ in (8) is tailored to

$$
\frac{d x}{d t}=\lambda W\left\{-N x+P_{\Omega}(D x+r)-q\right\}
$$

where $D=N-M$ and $r=q-p$. Similarly, if $F(x)=$ $M x+p$ in (19), then the GNCP becomes the generalized linear complementarity problem (GLCP), and the NN in (20) is tailored to

$$
\frac{d x}{d t}=\lambda W\left\{-N x+(D x+r)^{+}-q\right\}
$$

where $D=N-M$ and $r=q-p$.

All the stability results for the $\mathrm{NN}$ in (8) presented in Theorems 1-5 can be applied to the NNs in (20)-(22). For the latter two NNs, one point should be noted is that because $\nabla F(x)^{T} N$ and $\nabla F(x)^{T} \tilde{N}$ become $M^{T} N$ and $M^{T} \tilde{N}$, the conditions in these theorems requiring that the two matrices are positive definite or positive semidefinite on $\bar{\Omega}$ are equivalent to requiring that they are positive definite or positive semidefinite on the whole space. Another point should be noted is that the NN in (21) has been studied in [18] and [19] with different choices of $W$. Compared with the results therein, it is seen that Theorems 1-5 provide many alternative conditions for ascertaining the global convergence of this particular NN.

\section{NUMERICAL EXAMPLES}

In this section, we demonstrate the effectiveness and performance of the proposed NN by using several examples.

Example 1: Consider a GVI in (4) with

$$
\begin{gathered}
N=\left(\begin{array}{ll}
5 & 1 \\
2 & 3
\end{array}\right) \quad F(x)=N x+\left(\begin{array}{c}
\sin ^{2} x_{2}-3 \\
\cos \left(x_{1}+x_{2}\right)
\end{array}\right) \\
q=(-4-2)^{T} \quad \Omega=\left\{x \in R^{2} \mid 1 \leq x_{i} \leq 5, i=1,2\right\}
\end{gathered}
$$

It is easy to check that $U(x)=N x+q-F(x)$ is globally Lipschitz continuous with the Lipschitz constant $L=2$. Because $\mu_{\min }\left(N^{S}\right)=2.2>L$, in view of Theorem 1 , the NN in (8) with $W=I$, or the GPNN in (5) can be used to solve the problem. The trajectories of the NN with $\lambda=1$ started from 20 


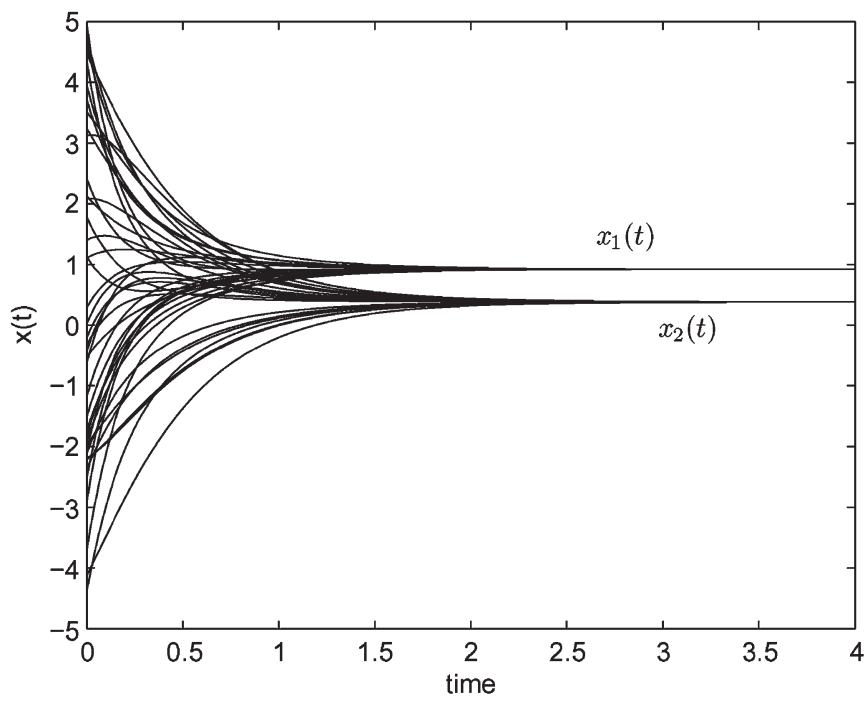

Fig. 2. Transient behavior of the GPNN in (5) with 20 random initial points in Example 1.

initial points are presented in Fig. 2, which shows that all trajectories converge to the unique solution $x^{*}=(0.923,0.385)^{T}$ exponentially.

Example 2: Consider a GVI in (7) with

$$
\begin{aligned}
F(x) & =\left(\begin{array}{c}
x_{2}+x_{3}-\exp \left(-x_{1}-x_{2}-x_{3}\right) \\
x_{1}+x_{3}-\exp \left(-x_{1}-x_{2}-x_{3}\right) \\
x_{1}+x_{2}-\exp \left(-x_{1}-x_{2}-x_{3}\right)
\end{array}\right) \\
N & =\left(\begin{array}{ccc}
-1 & 2 & 2 \\
2 & -1 & 2 \\
2 & 2 & -1
\end{array}\right) \quad q=\left(\begin{array}{c}
2 \\
-3 \\
5
\end{array}\right)
\end{aligned}
$$

and $\quad \Omega=\left\{x \in R^{3} \mid l \leq x \leq u\right\}, \quad l=(-5,-5,-5)^{T}, \quad u=$ $(0,10,5)^{T}$. It can be verified that the $\nabla F(x)+N$ is not positive semidefinite in $R^{3}$. As a result, the GPNN in (5) proposed in [18] cannot be ensured for its convergence and stability. In fact, simulations showed that the trajectory of this $\mathrm{NN}$ with any initial point always diverges to infinity (e.g., see Fig. 3). Now, we check the following conditions: $N$ is nonsingular, $\Omega$ is bounded, and

$$
\begin{aligned}
\nabla F & (x)^{T} N \\
\quad & =\left(\begin{array}{lll}
3 e^{-x_{1}-x_{2}-x_{3}}+4 & 3 e^{-x_{1}-x_{2}-x_{3}}+1 & 3 e^{-x_{1}-x_{2}-x_{3}}+1 \\
3 e^{-x_{1}-x_{2}-x_{3}}+1 & 3 e^{-x_{1}-x_{2}-x_{3}}+4 & 3 e^{-x_{1}-x_{2}-x_{3}}+1 \\
3 e^{-x_{1}-x_{2}-x_{3}}+1 & 3 e^{-x_{1}-x_{2}-x_{3}}+1 & 3 e^{-x_{1}-x_{2}-x_{3}}+4
\end{array}\right)
\end{aligned}
$$

is symmetric. Thus, the NN in (8) with $W=N^{-1}$ can be adopted to solve the problem. Simulation results showed that the trajectory of the $\mathrm{NN}$ started from any initial point converges to the unique solution $x^{*}=(0.765,-0.716,0.098)^{T}$, which is consisted with Theorem 2. Fig. 4 displays the transient behavior of the $\mathrm{NN}$ with 20 random initial points in $R^{3}$ when $\lambda=1$.

Example 3: Consider a GVI in (7) with

$$
\begin{aligned}
F(x) & =\left(\begin{array}{c}
-3\left(x_{1}+1\right)^{2}+10 \\
2\left(x_{2}-3\right)
\end{array}\right) \quad N=\left(\begin{array}{cc}
-1 & 0 \\
0 & 2
\end{array}\right) \\
q & =(1-2)^{T} \quad \Omega=\left\{x \in R^{2} \mid\left\|x-(1,1)^{T}\right\| \leq 1\right\} .
\end{aligned}
$$

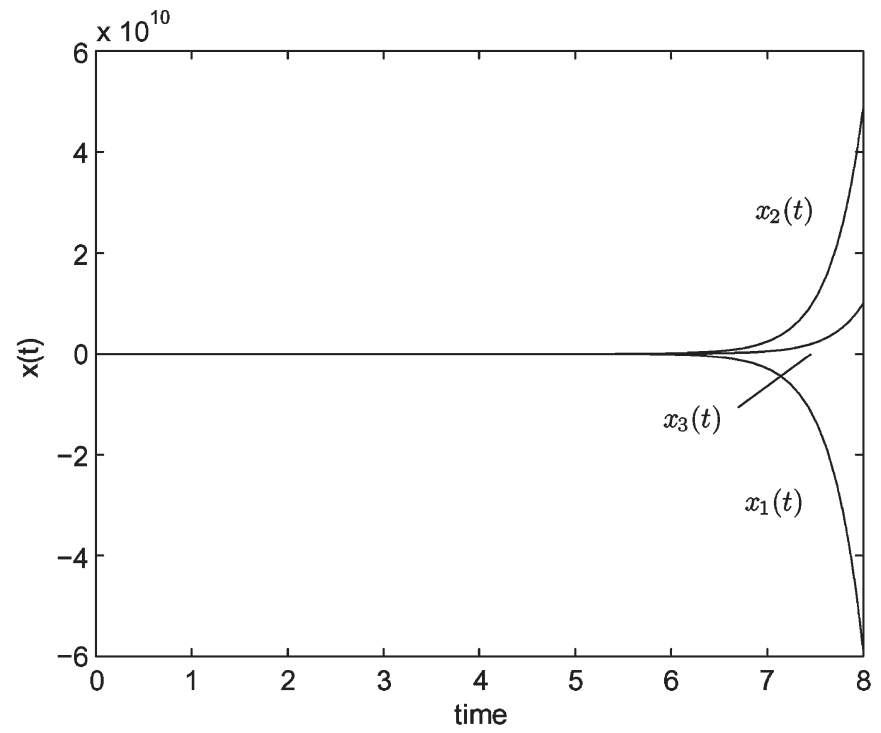

Fig. 3. Transient behavior of the GPNN in (5) with a random initial point in Example 2.

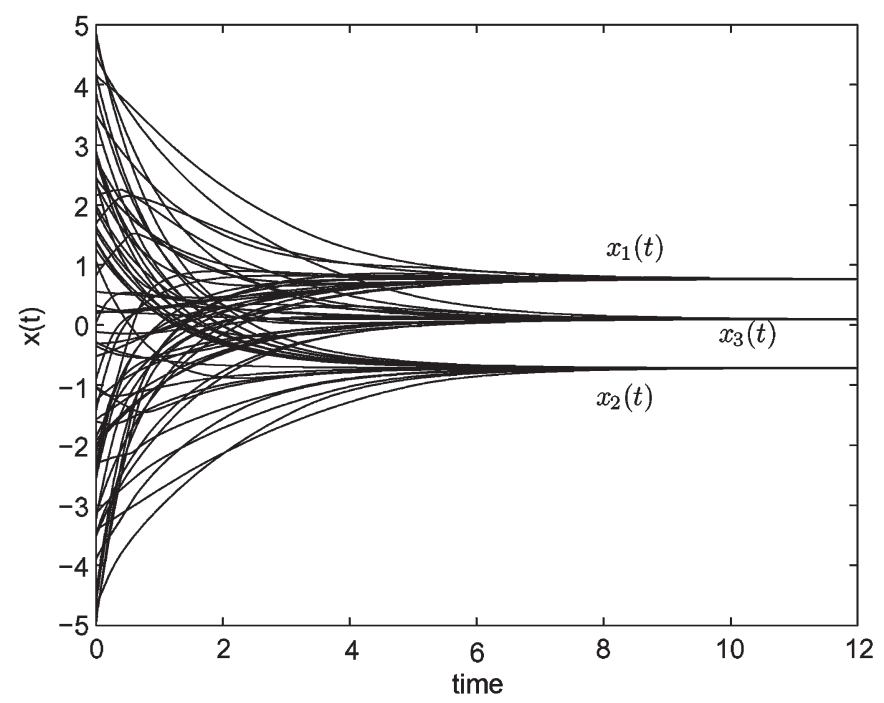

Fig. 4. Transient behavior of the NN in (8) with $W=N^{-1}$ and 20 random initial points in Example 2.

This example is used to show the effectiveness of the NN with $\Omega$ being a sphere instead of a box, and also to show some other results stated in Theorem 2. The Jacobian matrix of $F(x)$ is

$$
\nabla F(x)=\left(\begin{array}{cc}
-6\left(x_{1}+1\right) & 0 \\
0 & 2
\end{array}\right)
$$

Clearly, $\nabla F(x)+N$ is symmetric but not positive semidefinite in $R^{n}$ and the GPNN in (5) cannot be applied to solve the problem. However, it is trivial to show that the matrix

$$
\nabla F(x)^{T} N=\left(\begin{array}{cc}
6\left(x_{1}+1\right) & 0 \\
0 & 4
\end{array}\right)
$$

is symmetric and positive semidefinite for all $x$ satisfying $N x+$ $q \in \Omega$. According to Theorem 2, the NN in (8) with $W=N^{-1}$ will be globally convergent to the solution set of the problem. 


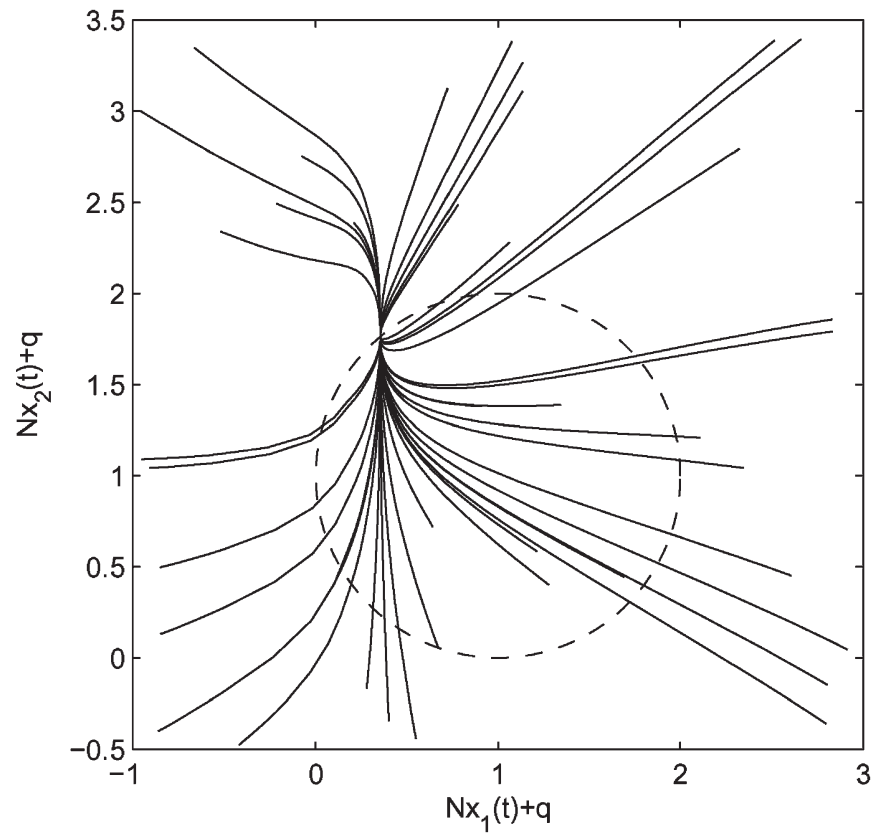

Fig. 5. Transient behavior of the NN in (8) with $W=N^{-1}$ and 40 random initial points in Example 3 when $q=(1,-2)^{T}$. The dashed circle stands for the boundary of $\Omega$.

The simulation results showed that the NN is globally asymptotically stable at the unique solution $x^{*}=(0.645,1.882)^{T}$. Fig. 5 displays the convergence of the $\mathrm{NN}$ with 40 random initial points in state space.

We next let $q=(2,-2)^{T}$. Then, $\nabla F(x)^{T} N$ becomes positive definite for all $x$ satisfying $N x+q \in \Omega$. According to Theorem 2, the $\mathrm{NN}$ in (8) should be globally exponentially stable. In Fig. 6, trajectories of the NN started from 30 random initial points are plotted, all of which converge exponentially to the solution $x^{*}=(0.853,1.995)^{T}$.

Example 4: Consider a GLVI in (4) with

$$
\begin{aligned}
M & =\left(\begin{array}{ccc}
-1 & 2 & 0 \\
0 & 3 & -3 \\
-1 & 0 & 1
\end{array}\right) \\
N & =\left(\begin{array}{ccc}
-3.5188 & 5.9858 & -0.2662 \\
0.2317 & 9.4938 & -9.1973 \\
-2.5387 & 0.3569 & 3.4999
\end{array}\right) \\
p & =(1,-3,-5)^{T} \quad q=(-5,0,-6)^{T}
\end{aligned}
$$

and $\Omega=\left\{x \in R^{4} \mid-5 \leq x_{i} \leq 5, i=1,2,3\right\}$. Note that $\operatorname{Rank}(N)=2$. By singular value decomposition, $N$ can be decomposed as $N=P \Lambda Q^{T}$, where $\Lambda=\operatorname{diag}(14.1695$, 6.4113,0), $P$ and $Q$ are two orthogonal matrices. Let $A=$ $\operatorname{diag}(14.1695,6.4113,1)$ and then

$$
\tilde{N}=P A Q^{T}=\left(\begin{array}{ccc}
-3.1169 & 6.2339 & 0 \\
0 & 9.3508 & -9.3508 \\
-3.1169 & 0 & 3.1169
\end{array}\right)
$$

It is verified that $M^{T} N$ is positive semidefinite and $M^{T} \tilde{N}$ is symmetric and positive definite. According to Theorem 3, the problem can be solved by using the $\mathrm{NN}$ in (8) with $W=\tilde{N}^{-1}$.

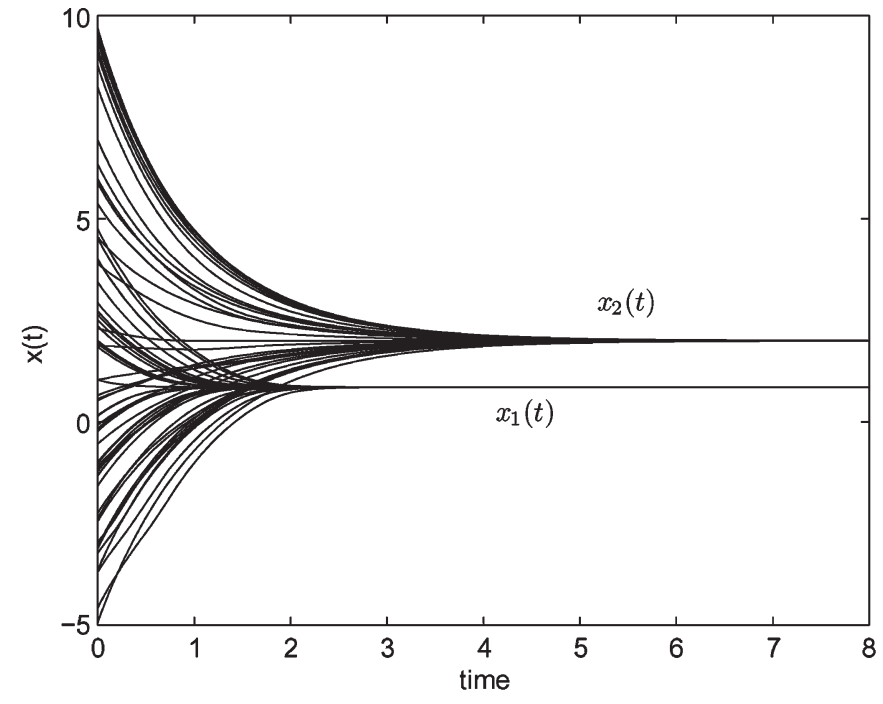

Fig. 6. Transient behavior of the NN in (8) with $W=N^{-1}$ and 30 random initial point in Example 3 when $q=(2,-2)^{T}$.

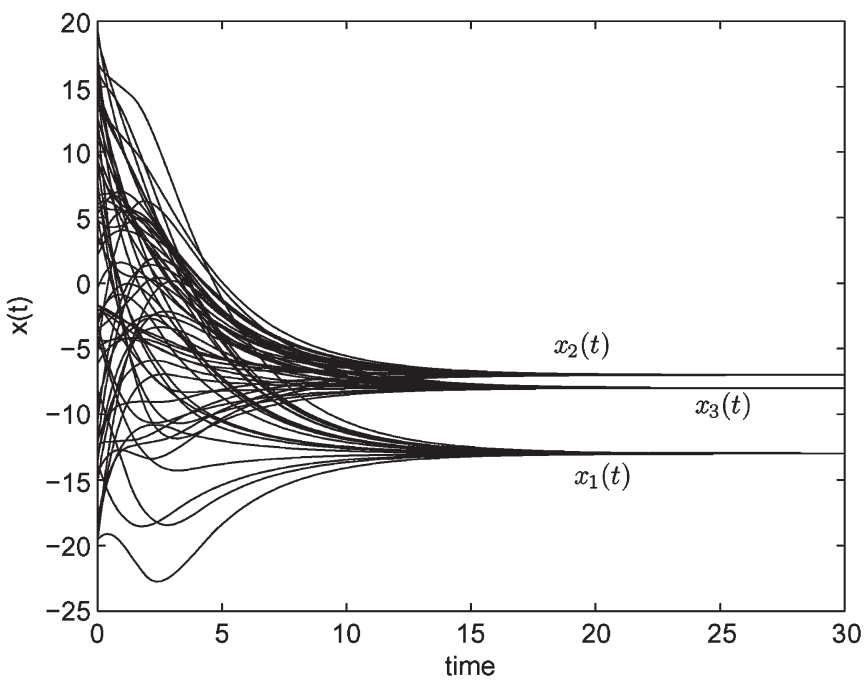

Fig. 7. Transient behavior of the NN in (8) with $W=\tilde{N}^{-1}$ and 20 random initial points in Example 4.

All simulations showed that the NN is globally convergent to the unique solution of the GLVI, $x^{*}=(-1.267,-0.100$, $-3.000,2.500)^{T}$. Fig. 7 illustrates the trajectories of the NN with 20 random initial points when $\lambda=1$. Simulation results also showed that if GPNN in (5) is used to solve the problem, the states always diverge.

Example 5: Consider a GLCP in (19) where $F(x)=M x+$ $p$ by using the $\mathrm{NN}$ in (22). Let

$$
\begin{aligned}
M & =\left(\begin{array}{ccccc}
5 & -3 & 0 & 0 & 2 \\
1 & 5 & 0 & 0 & 2 \\
3 & 0 & 2 & -2 & 0 \\
0 & 0 & 0 & 3 & 4 \\
-5 & -1 & 0 & 0 & -6
\end{array}\right) \quad p=\left(\begin{array}{c}
2 \\
1 \\
-2 \\
2 \\
4
\end{array}\right) \\
N & =\left(\begin{array}{ccccc}
4 & -3 & 0 & 0 & 0 \\
1 & 0 & 0 & 0 & 0 \\
0 & 0 & 0 & 0 & 0 \\
0 & 0 & 0 & 1 & 0 \\
0 & 0 & 0 & 1 & -1
\end{array}\right) \quad q=\left(\begin{array}{c}
1 \\
1 \\
3 \\
-5 \\
0
\end{array}\right) .
\end{aligned}
$$




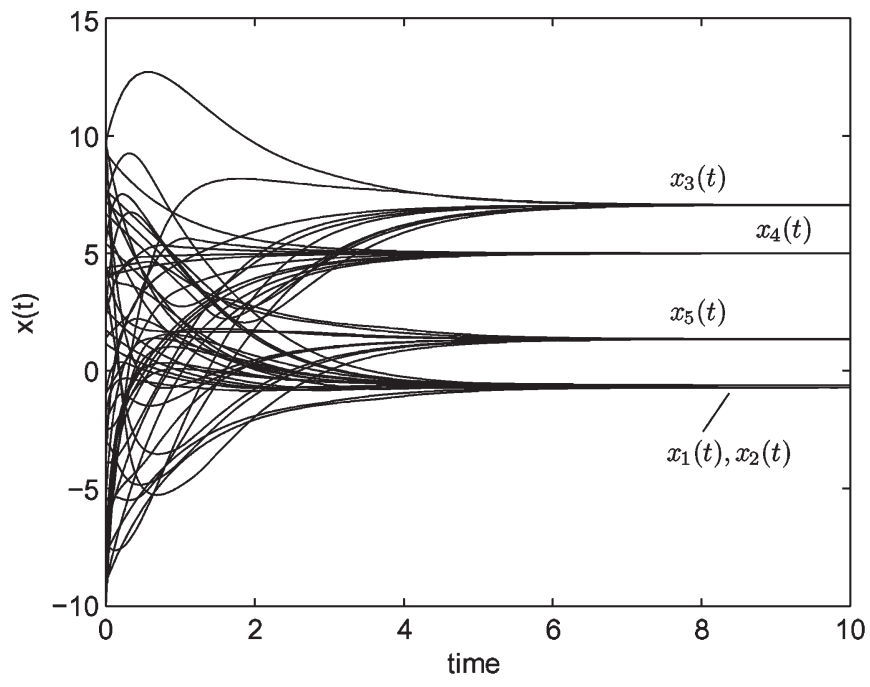

Fig. 8. Transient behavior of the NN in (22) with $W=\tilde{N}^{-1}$ and ten random initial points in Example 5.

The problem has a unique solution $x^{*}=(-0.700,-0.600$, $7.050,5.000,1.350)^{T}$. Note that $N$ is in the form of (18). According to Remark 5, the NN with $W=\tilde{N}^{-1}$ can be used to solve the problem, where $\tilde{N}$ is as same as $N$ except that the entry at the third row and third column is one instead of zero. It is verified that $M^{T} N$ is positive semidefinite and $M^{T} \tilde{N}$ is positive definite, i.e., two main conditions in Theorem 5 are satisfied. We use the NN to solve the problem. All simulations showed that the NN is globally convergent to $x^{*}$. Fig. 8 displays the state trajectories of the $\mathrm{NN}$ with ten random initial points when $\lambda=1$.

\section{CONCLUding REMARKS}

This paper presents a recurrent $\mathrm{NN}$ for solving a class of GVIs, which can be viewed as a modified version of the GPNN existing in the literature. Under mild conditions, it is shown that the proposed NN is globally convergent, globally asymptotically stable, and globally exponentially stable. Compared with the GPNN, the NN enlarges the scope of the GVIs that can be solved by recurrent NNs, while preserving the simple structure and low complexity for model implementation. This $\mathrm{NN}$ can be utilized to solve several special cases of this class of GVIs, GLVI, GLCP, and GNCP. The simulation results show the desirable performance of the proposed NN and substantiate the theoretical results.

\section{REFERENCES}

[1] A. Cichocki and R. Unbehauen, Neural Networks for Optimization and Signal Processing. Chichester, U.K.: Wiley, 1993.

[2] J. Wang, "Analysis and design of a recurrent neural network for linear programming," IEEE Trans. Circuits Syst. I, Fundam. Theory Appl., vol. 40, no. 9, pp. 613-618, Sep. 1993.

[3] X. Wu, Y. Xia, and W. Chen, "A high-performance neural network for solving linear and quadratic programming problems," IEEE Trans. Neural Netw., vol. 7, no. 3, pp. 643-651, May 1996.

[4] Y. G. Liu, Z. S. You, and L. P. Cao, "A functional neural network computing some eigenvalues and eigenvectors of a special real matrix," Neural Netw., vol. 18, no. 10, pp. 1293-1300, 2005.
[5] D. Kinderlehrer and G. Stampcchia, An Introduction to Variational Inequalities and Their Applications. New York: Academic, 1980.

[6] P. T. Harker and J. S. Pang, "Finite-dimensional variational inequality and nonlinear complementarity problems: A survey of theory, algorithms and applications," Math. Program., vol. 48, no. 2, pp. 161-220, 1990.

[7] F. Facchinei and J. S. Pang, Finite-Dimensional Variational Inequalities and Complementarity Problems. New York: Springer-Verlag, 2003.

[8] X. Liang and J. Si, "Global exponential stability of neural networks with globally Lipschitz continuous activations and its application to linear variational inequality problem," IEEE Trans. Neural Netw., vol. 12, no. 2, pp. 349-359, Mar. 2001.

[9] B. He and H. Yang, "A neural-network model for monotone linear asymmetric variational inequalities," IEEE Trans. Neural Netw., vol. 11, no. 1, pp. 3-16, Jan. 2000.

[10] Y. Xia and J. Wang, "On the stability of globally projected dynamical systems," J. Optim. Theory Appl., vol. 106, no. 1, pp. 129-150, 2000.

[11] Y. Xia, H. Leung, and J. Wang, "A projection neural network and its application to constrained optimization problems," IEEE Trans. Circuits Syst. I, Fundam. Theory Appl., vol. 49, no. 4, pp. 447-458, Apr. 2002.

[12] Y. Xia, "Further results on global convergence and stability of globally projected dynamical systems," J. Optim. Theory Appl., vol. 122, no. 3, pp. 627-649, Sep. 2004.

[13] X. Hu and J. Wang, "Solving pseudomonotone variational inequalities and pseudoconvex optimization problems using the projection neural network," IEEE Trans. Neural Netw., vol. 17, no. 6, pp. 1487-1499, Nov. 2006.

[14] Y. Xia, "An extended projection neural network for constrained optimization," Neural Comput., vol. 16, no. 4, pp. 863-883, 2004.

[15] Y. Xia and G. Feng, "On convergence conditions of an extended projection neural network," Neural Comput., vol. 17, no. 3, pp. 515-525, 2005.

[16] X. Gao, L. Liao, and L. Qi, "A novel neural network for variational inequalities with linear and nonlinear constraints," IEEE Trans. Neural Netw., vol. 16, no. 6, pp. 1305-1317, Nov. 2005.

[17] M. A. Noor, "General variational inequalities," Appl. Math. Lett., vol. 1, no. 2, pp. 119-121, 1988

[18] Y. Xia and J. Wang, "A general projection neural network for solving monotone variational inequalities and related optimization problems," IEEE Trans. Neural Netw., vol. 15, no. 2, pp. 318-328, Mar. 2004.

[19] X. Gao, "A neural network for a class of extended linear variational inequalities," Chin. J. Electron., vol. 10, no. 4, pp. 471-475, 2001.

[20] J. S. Pang and J. C. Yao, "On a generalization of a normal map and equations," SIAM J. Control Optim., vol. 33, no. 1, pp. 168-184, 1995.

[21] A. Auslender, Optimisation: Méthodes Numériques. Paris, France: Masson, 1976.

[22] G. Mastroeni, "Gap functions and descent methods for Minty variational inequality," in Optimization and Control With Applications. Applied Optimization, vol. 96, L. Qi, K. Teo, and X. Yang, Eds. New York: SpringerVerlag, 2005, pp. 529-547.

[23] M. Fukushima, "Equivalent differentiable optimization problems and descent methods for asymmetric variational inequality problems," Math. Program., vol. 53, no. 1, pp. 99-110, 1992.

[24] J. J. Slotine and W. Li, Applied Nonlinear Control. Englewood Cliffs, NJ: Prentice-Hall, 1991.

[25] S. Grossberg, "Nonlinear difference-differential equations in prediction and learning theory," Proc. Nat. Acad. Sci., vol. 58, no. 4, pp. 1329-1334, 1967.

[26] - "Global ratio limit theorems for some nonlinear functional differential equations I," Bull. Amer. Math. Soc., vol. 74, no. 1, pp. 95-99, 1968.

[27] _ "Global ratio limit theorems for some nonlinear functional differential equations II," Bull. Amer. Math. Soc., vol. 74, no. 1, pp. 100-105, 1968.

[28] J. J. Hopfield, "Neurons with graded response have collective computational properties like those of two-state neurons," Proc. Nat. Acad. Sci., vol. 81, no. 10, pp. 3088-3092, 1984.

[29] Z. Yi and K. K. Tan, Convergence Analysis of Recurrent Neural Networks. Boston, MA: Kluwer, 2004.

[30] Z. Yi, M. Ye, J. C. Lv et al., "Convergence analysis of a deterministic discrete time system of Oja's PCA learning algorithm," IEEE Trans. Neural Netw., vol. 16, no. 6, pp 1318-1328, Nov. 2005.

[31] Z. Zeng and J. Wang, "Improved conditions for global exponential stability of recurrent neural networks with time-varying delays," IEEE Trans. Neural Netw., vol. 17, no. 3, pp. 623-635, May 2006.

[32] — "Complete stability of cellular neural networks with timevarying delays," IEEE Trans. Circuits Syst. I, Reg. Papers, vol. 53, no. 4, pp. 944-955, Apr. 2006.

[33] B. He, "Solution and applications of a class of general linear variational inequalities," Sci. China Ser. A-Math., vol. 39, no. 25, pp. 397-404, 1996. 


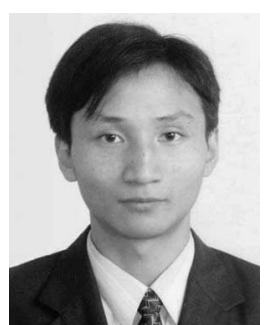

Xiaolin Hu (S'01) received the B.E. and M.E. degrees in automotive engineering from the Wuhan University of Technology, Wuhan, China, in 2001 and 2004 , respectively. He is currently working toward the Ph.D. degree at the Chinese University of Hong Kong, Sha Tin.

His current research interests include neural networks and their applications in intelligent control.

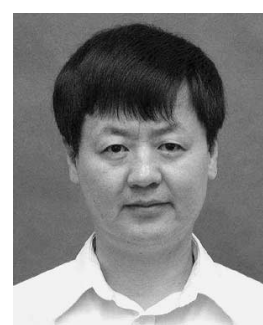

Jun Wang (S'89-M'90-SM'93-F'07) received the B.S. degree in electrical engineering and the M.S. degree in systems engineering from Dalian University of Technology, Dalian, China, in 1982 and 1985, respectively, and the Ph.D. degree in systems engineering from Case Western Reserve University, Cleveland, OH, in 1991.

He was an Associate Professor with the University of North Dakota, Grand Forks, until 1995. He is currently a Professor with the Department of Automation and Computer-Aided Engineering, Chinese University of Hong Kong, Sha Tin. His current research interests include neural networks and their engineering applications.

Dr. Wang is an Associate Editor of the IEEE TRANSACTIONS ON Neural Networks and IEEE Transactions on Systems, Man, and Cybernetics: PART B. He was the President of the Asia Pacific Neural Network Assembly. 